\title{
Avaliação de Repositórios de Objetos de Aprendizagem com Métodos multicritérios baseada em Indicadores de Qualidade
}

\author{
Rosymeire Oliveira da Silva $^{1}$, Adriano Fiorese ${ }^{1}$ \\ ${ }^{1}$ Programa de Pós-Graduação em Computação Aplicadada (PPGCA) \\ Departamento de Ciência da Computação (DCC) \\ Universidade do Estado de Santa Catarina (UDESC) - Joinville - SC - Brazil \\ rosymeireoliveira26@gmail.com, adriano.fiorese@udesc.br
}

\begin{abstract}
Learning Object Repositories (LOR), are appropriate platforms for the storage of Learning Objects (LO). They make it easier for educational resources to be found and reused. However, the wide variety of LORs and resources available makes it difficult for the user to find an LOR that is appropriate in terms of their needs. In this sense, this work presents an ROA evaluation method that aims to offer the most suitable ones according to the user's needs. The proposed method uses the Analytic Hierarchy Process (AHP) which aims, at the end, to rank the most suitable alternatives according to the user's requests due to a required set of Indicators and their values
\end{abstract}

Resumo. Repositórios de Objetos de Aprendizagem (ROA), são plataformas apropriadas para o armazenamento de Objetos de Aprendizagem (OAs). Eles facilitam que os recursos educacionais possam ser encontrados e reutilizados. Porém, a grande variedade de ROAs e recursos disponíveis, dificulta ao usuário encontrar um ROA que seja adequado em termos de suas necessidades. Nesse sentido, este trabalho apresenta um método para avaliação de ROAs que visa oferecer as melhores alternativas de acordo com as necessidades do usuário. O método proposto faz uso do Analytic Hierarchy Process (AHP) que visa, ao final, ranquear as mais adequadas alternativas de acordo com as requisições do usuário em virtude de um conjunto requerido de Indicadores e seus valores.

\section{Introdução}

Com o crescimento do e-learning, a busca por recursos educacionais digitais, tem se tornado cada vez mais necessária para o processo de diversificação de estratégias de aprendizado. Dessa forma, é importante que esses recursos estejam armazenados em espaços apropriados e que facilitem a busca e seleção para reutilização em variados ambientes de aprendizagem. Para isso Repositórios de Objetos de Aprendizagem (ROAs) tratam-se de espaços específicos para o armazenamento desses recursos de aprendizagem.

Porém, o grande crescimento da produção de objetos de aprendizagem, especialmente envolvendo componentes pedagógicos, tecnológicos e colaborativos, se torna proporcional à oferta de uma grande demanda por repositórios, principalmente para $\mathrm{o}$ adequado armazenamento, disponibilidade e posteriormente a reutilização dos OAs. Isso faz com que o usuário depare-se com uma grande quantidade de ROAs e OAs, tornando a escolha manual do mais adequado, em termos dos OA disponíveis e suas características, uma tarefa difícil. Nesse sentido, a avaliação manual ou humanamente comparativa dos 
ROAs, é eficiente até certo ponto. Entretanto, torna-se inviável a partir do momento que o número de recursos de aprendizagem armazenados nos ROAs aumenta mais rapidamente do que a capacidade do usuário de avaliá-los [Cechinel et al. 2016]. Dessa forma, torna-se evidente a necessidade de uma forma automatizada para tal.

Pensando nisso, este trabalho apresenta um método de avaliação de ROAs baseado em um método multicritério de tomada de decisão conhecido por Processo Hierárquico Analítico (AHP, do inglês Analytic Hierarchy Process) [Saaty 1980]. Tal método tem por objetivo realizar a análise dos ROAs observando a relação entre a meta de escolha do mais adequado ROA e os critérios para tal escolha (que todos os ROAs devem possuir). A essas relações é também acrescentada a necessidade do usuário relativa a utilização do ROA, modelada por meio de uma requisição envolvendo todos ou parte dos critérios utilizados na análise. Como resultado dessa análise, o método proposto devolve ao usuário uma lista ordenada do mais adequado ao menos adequado ROA. Nesse sentido, como critérios de avaliação são utilizados indicadores baseados na perspectiva de avaliação da qualidade de software da International Organization for Standardization (ISO) de acordo com a norma 25010 (ISO 25010), que são: Funcionalidade, Usabilidade, Compatibilidade e Confiabilidade [Standardization 2016].

As próximas seções do trabalho estão organizadas da seguinte forma: A Seção 2 apresenta os conceitos necessários para a compreensão do trabalho, envolvendo ROA, AHP e os indicadores de qualidade. A Seção 3 aborda trabalhos relacionados que envolvem avaliações realizadas em ROAs. A Seção 4 apresenta e discute em detalhes o método proposto de avaliação de ROAs. A Seção 5 apresenta os experimentos e análises dos resultados obtidos referentes a avaliação de 10 ROAs reais e finalmente, a Seção 6 apresenta as considerações finais e o que se espera como trabalhos futuros.

\section{Fundamentação Teórica}

Esta seção apresenta os principais conceitos que envolvem o desenvolvimento deste trabalho, e que são necessários a sua compreensão.

\subsection{Repositórios de Objetos de Aprendizagem}

Repositórios de Objetos de Aprendizagem são softwares educacionais que permitem o armazenamento, pesquisa e a reutilização de objetos de aprendizagem. Sua importância caracteriza-se pelo fato de armazenar e portanto disponibilizar vários recursos educacionais que facilitam o ensino e motivam o aprendizado. De acordo com [Braga 2015], um ROA deve permitir: O depósito de conteúdo pelo criador, proprietário ou por um terceiro; Possuir arquitetura capaz de administrar conteúdos e metadados; Oferecer serviços para armazenar, retirar e buscar recursos, além de serviços de controle de acesso aos mesmos; Ser confiável, bem suportado e bem administrado. Geralmente ROAs são mantidos por entidades governamentais, o que faz com que a segurança e a persistência do seu link sejam adequadas ao seu reúso.

Os recursos que os ROAs disponibilizam são os Objetos de Aprendizagem (OA). Tal conceito surgiu no final do ano 1990 e define-se, segundo, a IEEE [Committee et al. 2002], como qualquer entidade, digital ou não digital que pode ser usada, reutilizada ou referenciada durante o aprendizado. Neste trabalho assume-se somente OAs digitais. Para que esses Recursos (OA) sejam facilmente localizados e recuperados é de grande importância algum padrão de metadados com todas as suas informações 
IX Congresso Brasileiro de Informática na Educação (CBIE 2020)

Anais do XXXI Simpósio Brasileiro de Informática na Educação (SBIE 2020)

preenchidas, pois as informações contidas nos mesmos definirão a qualidade do objeto, sua interoperabilidade e a possibilidade de sua reutilização.

\subsection{Analytic Hierarchy Process}

O Analytic Hierarchy Process (AHP) é um método multicritério de tomada de decisão, desenvolvido por Thomas L. Saaty [Saaty 1980]. Ele é muito eficaz para lidar com decisões complexas, pois estabelece a necessidade de níveis de prioridade entre os critérios utilizados para a solução, e entre critérios e alternativas de solução. Essa organização visa realizar uma avaliação pareada entre os envolvidos com vistas a determinar um resultado consistente a respeito da melhor alternativa de solução ao problema proposto.

O AHP usa um conjunto padrão de valores inteiros (1-9), conhecido como escala de Saaty [Saaty 1980], para descrever a importância da relação entre os níveis hierárquicos, onde 9 representa a maior importância de um elemento (critério ou alternativa) em relação ao outro, e 1 é a menor importância. O número 1 também significa que os critérios sendo avaliados são igualmente importantes entre si. A escala de Saaty é usada nas matrizes de comparação, também conhecidas por matrizes de julgamento, entre critério e objetivo e entre critérios e alternativas, e seus valores basicamente representam o grau de importância que um item tem em relação ao outro. Após esse processo procede-se à pontuação final (global) de cada alternativa em relação ao objetivo.

\section{Trabalhos Relacionados}

Para escolher um ROA com significativa acessibilidade e com qualidade para a educação, se faz necessária a aplicação de um modelo ou método que seja conveniente, isto é, apropriado para fazer tal avaliação [Kurilovas 2013], sem que exija do usuário grande predisposição na comparação e disposição de tempo. Levando em conta essa consideração, os métodos multicritério de tomada de decisão (MCDM), são de grande eficácia para realizar uma avaliação consistente entre os ROA, de forma que auxiliem o usuário a tomar decisões precisas sobre quais ROAs podem ser os mais adequados.

Nesse sentido, [Yigit et al. 2014] realizaram uma abordagem utilizando o método multi-critério para auxílio a tomada de decisão "Analytic Hierarchy Process"(AHP) com intuito principal de reduzir o desperdício de tempo e aprimorar a precisão da tomada de decisões utilizando os metadados dos objetos de aprendizagem. Os autores desenvolveram o ROA chamado SDUNESA [Yigit et al. 2014] utilizado para armazenar e compartilhar OAs e seus metadados. Posteriormente os autores [İnce et al. 2019] realizaram outro trabalho de avaliação e seleção de OAs em ROA utilizando o método AHP combinado com algoritmo genético(GA). Nesse trabalho foi utilizada uma abordagem híbrida com o método AHP para o cálculo dos pesos utilizados na função de fitness do algoritmo genético. O trabalho busca avaliar, selecionar e recomendar OAs apropriados de acordo com as preferências e parâmetros da busca realizada pelo usuário.

No trabalho de [Kurilovas 2013], os autores criaram o método MCEQLS, do inglês (Approach in MultiCriteria Evaluation of Quality of Learning Repositories) com objetivo de melhorar a qualidade da avaliação especializada do software de aprendizagem. Essa ferramenta tem como primeiro passo a identificação de critérios que podem ser qualitativos e quantitativos. Os critérios levantados e justificados pelos autores são os seguintes: Navegação; Compartilhamento e aquisição de OA; Página de metadados; 
Página inicial do ROA; Manter os usuários atualizados; Conta pessoal; Editores de metadados; Implementação de metadados gerado por usuário; Armazenamento de metadados descritivos. Esses critérios também foram utilizados em uma pesquisa posterior para aprimoração do MCEQLS chamada NEWS MCEQLS [Kurilovas et al. 2016], para avaliar as funcionalidades dos ROAs e a sua qualidade de uso. Para isso foi utilizado o método híbrido AHP-Fuzzy.

Em vista dos trabalhos apresentados, pode-se observar que características fundamentais como armazenamento, navegabilidade, metadados, entre outras, são importantes para que o ROA seja considerado de qualidade para o usuário. Assim, para realizar a avaliação dos ROAs, tais caracterısticas podem ser aplicadas aos métodos multicritérios, como por exemplo o AHP. No presente trabalho, além de se utilizar tais características fundamentais, que são baseadas nos indicadores da norma de qualidade ISO 25010, diferencialmente, também avalia-se o atendimento da requisição do usuário, com o objetivo de fornecer os mais adequados ROAS.

\section{Método Proposto}

Esta seção apresenta e discute o método proposto de avaliação de ROAs. Ele é baseado no método de tomada de decisão multicritério Analytic Hierarchy Process (AHP), que modela a solução do problema calcada na hierarquização do objetivo final, critérios para a tomada de decisão e alternativas de solução. Também leva em consideração o atendimento ou não da necessidade do usuário, tratada como uma requisição, que contém valores para os critérios desejados.

A Figura 1 representa a arquitetura do método de avaliação de ROAs proposto. Assim, o módulo matriz de julgamento é onde se realiza a avaliação por parte de especialistas, da relação entre indicadores/critérios, de forma pareada, com a finalidade de estabelecer a importância relativa entre eles em função do objetivo do problema. Tal avaliação busca encontrar os respectivos pesos dos indicadores/critérios utilizados. Tais pesos serão utilizados no método proposto para calcular um valor de pontuação final para cada alternativa.

A base de dados da arquitetura contém os indicadores utilizados, com seus respectivos critérios e características essenciais dos ROAs, por meio de valores que os compõem. Tais valores são obtidos pelo módulo Inspeção nos ROAs, que trata da coleta direta e de forma não automatizada dos mesmos junto aos ROAs.

No módulo de requisição, o usuário (assumindo que seja o usuário final) irá solicitar em relação aos ROAs, os recursos que lhe convém. Por sua vez, o módulo AHP executará o método AHP, utilizando-se dos critérios e seus valores da base de dados, dos pesos para esses critérios obtidos pelas matrizes de julgamento fornecidas pelos especialistas e pela requisição do usuário final. Ao ser executado o AHP, os ROAs receberão sua pontuação final, que é utilizada para o ranqueamento/ordenação final, de forma decrescente. Para o caso de haver mais de um ROA classificado com os mesmos valores de pontuação, prevalece o ROA com o maior valor envolvendo a soma dos seus indicadores.

\subsection{Indicadores selecionados e seus pesos}

Para atribuir valores aos Indicadores derivados da norma ISO 25010 utilizados como critérios de tomada de decisão, foi necessária uma coleta de informações junto aos ROAs, 


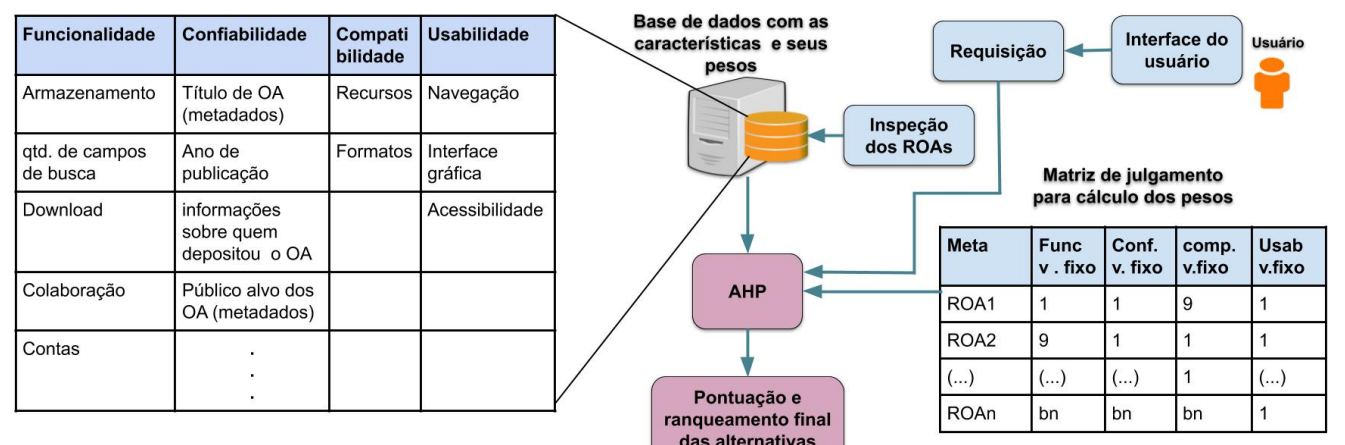

Figura 1. Arquitetura do Método de Avaliação de ROAs

contemplando os critérios/características individuais que os compõem. Os Indicadores, seus critérios e a definição dos seus valores deu-se da seguinte forma: Funcionalidade: Este Indicador é composto pelas características: quantidade de formas nas quais o usuário pode armazenar $(A r)$ seus recursos educacionais; a quantidade de mecanismos de busca $(M b)$ oferecidos para ajudar o usuário encontrar os recursos de forma ágil; quantidade de meios de colaboração $(C l)$ e conta particular $(C p)$ para gerenciamento dos recursos (1 se o ROA disponibiliza, 0 caso contrário). Confiabilidade: Este indicador é composto pela quantidade de campos de metadados $(M e)$ dos objetos de aprendizagem, disponibilizados pelo ROA. Compatibilidade: Este Indicador tem a ver com a quantidade de tipo de recursos $(R e)$ e formatos $\left(F_{O}\right)$ de objetos de aprendizagem que o ROA disponibiliza. Usabilidade: Indicador cujo propósito é a otimização da experiência do usuário. Para o cálculo do valor desse Indicador, leva-se em conta a navegabilidade $(\mathrm{Na})$ dos ROAs, quantificando (0/1) se os mesmos possuem formulário de pesquisa simples e avançado, se lista os resultados das pesquisas e etc; a interface gráfica $(I n)$, quantificando se o ROA mantém o objetivo central, deixando explícita sua área temática, atualização sobre atividades, boletins ou informativos; bem como a Acessibilidade $(A c)$ que quantifica o número de opções de acesso disponibilizadas pelo ROA para usuários que tenham alguma deficiência.

Os pesos para os Indicadores utilizados resultam de um processo de avaliação pareada realizado por 4 especialistas, experientes em ROAs ou sistemas $e$-learning. Assim, cada especialista avaliou a importância para o objetivo de classificar o mais adequado ROA utilizando tais Indicadores, por meio de uma matriz de julgamento. A Tabela 1 apresenta os valores (utilizando a escala de Saaty) atribuídos pelos especialistas em avaliação pareada Indicador x Indicador. Ela apresenta para cada Indicador (linhas da tabela), o valor do peso (coluna Pesos) resultante para cada especialista. Ao final do processo, foi utilizada a média aritmética da avaliação dos 4 especialistas, resultando no seguinte conjunto de pesos: Funcionalidade: 0,23072, Usabilidade: 0,15003, Confiabilidade: 0,52831 e Compatibilidade: 0,09091.

\subsection{Atendimento da Requisição}

Assim, após encontrar os pesos para os Indicadores, o próximo passo é compatibilizar o atendimento da requisição do usuário pelos ROAs. O conceito de atendimento das requisições do usuário modela se o ROA consegue satisfazer o usuário utilizando uma abordagem quantitativa que expressa resultados qualitativos. Dessa forma, a Equação (1) 
IX Congresso Brasileiro de Informática na Educação (CBIE 2020)

Anais do XXXI Simpósio Brasileiro de Informática na Educação (SBIE 2020)

Tabela 1. Avaliação dos Especialistas

\begin{tabular}{|l|l|l|l|l|l|l|}
\hline Critério & Especialista & Func. & Usab. & Conf & Comp. & Pesos \\
\hline \multirow{5}{*}{ Func. } & Especialista 1 & 1 & 0,142 & 0,111 & 5 & 0,09565 \\
& Especialista 2 & 1 & 8 & 0,111 & 8 & 0,23249 \\
& Especialista 3 & 1 & 8 & 0,111 & 8 & 0,23249 \\
& Especialista 4 & 1 & 9 & 0,111 & 7 & 0,36226 \\
\hline \multirow{5}{*}{ Usab. } & Especialista1 & 7 & 1 & 0,111 & 7 & 0,22642 \\
& Especialista 2 & 0,125 & 1 & 0,111 & 8 & 0,11459 \\
& Especialista 3 & 0,125 & 1 & 0,111 & 8 & 0,11459 \\
& Especialista 4 & 0,111 & 1 & 9 & 0,125 & 0,14453 \\
\hline \multirow{5}{*}{ Conf. } & Especialista 1 & 9 & 9 & 1 & 9 & 0,63933 \\
& Especialista 2 & 9 & 9 & 1 & 9 & 0,61768 \\
& Especialista 3 & 9 & 9 & 1 & 9 & 0,61768 \\
& Especialista 4 & 9 & 0,111 & 1 & 0,125 & 0,23855 \\
\hline \multirow{5}{*}{ Comp. } & Especialista 1 & 0,2 & 0,142 & 0,111 & 1 & 0,03857 \\
& Especialista 2 & 0,125 & 0125 & 1 & 1 & 0,03522 \\
& Especialista 3 & 0,125 & 0,125 & 0,111 & 1 & 0,03522 \\
& Especialista 4 & 0,142 & 8 & 8 & 1 & 0,25464 \\
\hline
\end{tabular}

representa a função de atendimento $\left(\right.$ Atend) acerca do valor $\left(V_{i}\right)$ da requisição $(R q)$ em relação ao valor do critério $\left(C_{n}\right) \in$ ao Indicador $(I n d)$ de cada alternativa $\left(A_{j}\right)$. Assim, se o valor da requisição $\left(R q\left(V_{i}\right)\right)$ for maior que o valor do critério $\left(C_{n}\right)$ da alternativa $\left(A_{j}\right)$ então a alternativa $\left(A_{j}\right)$ não atende a requisição, e o valor da função (Atend) é igual a 1. Caso a requisição $\left(R q\left(V_{i}\right)\right)$ seja menor ou igual ao valor do critério $\left(C_{n}\right)$ da alternativa $\left(A_{j}\right)$, então a alternativa $\left(A_{j}\right)$ atende a requisição com valor igual a 9.

$$
\operatorname{Atend}\left(R q\left(V_{i}\right), C_{n}, A_{j}\right)=\left\{\begin{array}{l}
1, R q\left(V_{i}\right)>C_{n}\left(I_{n d}\right) \in A_{j} \\
9, R q\left(V_{i}\right) \leq C_{n}\left(I_{n} d_{n}\right) \in A_{j}
\end{array}\right.
$$

Esses valores são utilizados em uma matriz de julgamento pareada entre os ROAs para cada critério, que formam os Indicadores, envolvidos na requisição. Isso se repete para cada requisição do usuário. Assim, ao final da avaliação obtêm-se os valores de peso para cada valor de critério envolvido, conforme atende ou não à requisição do usuário, para cada uma das alternativas. Esses valores estão representados pela matriz $A_{j}^{i}$, na Equação(2), que apresenta o valor final da pontuação de cada alternativa.

\subsection{Classificação}

Com a posse dos valores dos critérios $C_{j}$, dos pesos dos critérios conforme atendimento da requisição do usuário $A_{j}^{i}$ e dos valores dos pesos dos critérios $P C_{j}$, de acordo com sua importância para o ranqueamento dos ROAs (objetivo) ${ }^{1}$, obtêm-se o valor final de pontuação pelo AHP. Esse valor é também chamado de pontuação global para cada Alternativa ${ }_{i}$, em relação ao objetivo, conforme Equação refeq:alternativaa). Onde $i=1,2, . ., n$ é o número total de alternativas e $j=1,2, \ldots, m$ é o número total de critérios.

\footnotetext{
${ }^{1}$ Os critérios assumem o peso correspondente do Indicador que os agrega. Esse peso é calculado com a ajuda dos especialistas, conforme discutido anteriormente.
} 


$$
\text { Alternativa }_{i}=\sum_{j=1}^{m} P C_{j} *\left(A_{j}^{i} * C_{j}\right)
$$

\section{Experimentos e Resultados}

Os valores presentes na base de dados servirão ao propósito de validação do método proposto, pois determinam os critérios essenciais levantados pela análise individual dos ROAs que serão utilizados nos experimentos. Cada coluna representa um critério pertencente aos seus respectivos Indicadores e o seu valor coletado dos ROAs. A Tabela 2 apresenta a base de dados mencionada, ilustrando os valores dos Indicadores utilizados no experimento, bem como a indicação de quais critérios os compõem.

Para validar a avaliação realizada pelo método proposto foram realizadas três requisições hipotéticas em um conjunto de 40 ROAs $^{2}$ reais. Na primeira requisição os valores para os critérios são os menores de forma a simular o caso em que a maioria dos ROAs os conseguem atender, definido como o melhor caso para o método. Na segunda requisição é esperado que o número de ROAs que atendam ao usuário reduza, uma vez que os recursos solicitados aumenta. Esse é o caso médio. E por fim, a última requisição, simula o pior caso, no qual o método encontra maior dificuldade para encontrar ROAs que atendam ao maior volume possível de recursos (critérios) para serem atendidos.

Tabela 2. Valores dos critérios associados aos ROAS, disponíveis na base de dados

\begin{tabular}{c|cccc|c|cc|ccc|c}
\hline \multirow{2}{*}{ ROAs } & \multicolumn{3}{|c|}{ Funcionalidade } & Confiabilidade & \multicolumn{3}{|c|}{ Compatibilidade } & \multicolumn{3}{|c}{ Usabilidade } & Total \\
& $A r$, & $M b$, & $C p$, & $C l$, & $M e$, & $R e$ & $F o$ & $N a$, & Int, & $A c$ & \\
\hline ROA1 & 2 & 4 & 4 & 1 & 6 & 11 & 3 & 13 & 8 & 2 & $\mathbf{5 4}$ \\
ROA3 & 2 & 4 & 3 & 4 & 11 & 8 & 3 & 14 & 5 & 0 & $\mathbf{5 4}$ \\
ROA4 & 2 & 7 & 3 & 1 & 11 & 3 & 3 & 15 & 8 & 1 & $\mathbf{5 4}$ \\
ROA5 & 2 & 3 & 3 & 5 & 9 & 6 & 3 & 11 & 6 & 2 & $\mathbf{5 0}$ \\
ROA6 & 3 & 7 & 3 & 4 & 11 & 6 & 5 & 14 & 5 & 1 & $\mathbf{5 9}$ \\
ROA14 & 2 & 4 & 2 & 9 & 8 & 7 & 3 & 10 & 6 & 1 & $\mathbf{5 2}$ \\
ROA16 & 0 & 2 & 2 & 5 & 7 & 3 & 2 & 11 & 5 & 1 & $\mathbf{3 8}$ \\
ROA25 & 3 & 9 & 2 & 8 & 12 & 13 & 6 & 11 & 8 & 6 & $\mathbf{7 0}$ \\
ROA27 & 1 & 5 & 2 & 6 & 6 & 9 & 4 & 8 & 6 & 2 & $\mathbf{4 9}$ \\
ROA28 & 2 & 8 & 2 & 7 & 12 & 11 & 3 & 16 & 8 & 2 & $\mathbf{7 1}$ \\
ROA30 & 2 & 6 & 0 & 7 & 9 & 10 & 6 & 17 & 3 & 1 & $\mathbf{6 1}$ \\
ROA37 & 1 & 6 & 0 & 7 & 12 & 17 & 6 & 13 & 8 & 1 & $\mathbf{7 1}$ \\
ROA38 & 4 & 1 & 2 & 8 & 7 & 8 & 1 & 6 & 7 & 0 & $\mathbf{4 4}$ \\
ROA39 & 2 & 4 & 1 & 5 & 14 & 9 & 4 & 4 & 4 & 0 & $\mathbf{4 7}$ \\
ROA40 & 2 & 4 & 2 & 9 & 8 & 7 & 4 & 13 & 5 & 2 & $\mathbf{5 6}$ \\
\hline
\end{tabular}

Assim, na primeira requisição, simulou-se a requisição por parte do usuário de 4 tipos de recursos $(r e q R e=4), 3$ tipos de formato $(r e q F o=3$ ), formas colaborativas ( $r e q C o$ $=3$ ) e informações descritas nos metadados $($ reqMe $=8)$. Para a segunda e terceira, as quantidades requisitadas aumentaram para cada um desses critérios. 
IX Congresso Brasileiro de Informática na Educação (CBIE 2020)

Anais do XXXI Simpósio Brasileiro de Informática na Educação (SBIE 2020)

Tabela 3. Dados para a primeira requisição e pontuação com AHP

\begin{tabular}{|c|c|c|c|c|c|}
\hline \multirow{2}{*}{ ROAs } & $\mathrm{Re}$ & $\mathrm{Fo}$ & $\mathrm{Cl}$ & $\mathrm{Me}$ & \multirow{2}{*}{ Pontuação } \\
\cline { 2 - 5 } & 0,09091 & 0,09091 & 0,23072 & 0,52831 & \\
\hline ROA25 & 0,082638 & 0,164647 & 0,109967 & 0,092222 & 0,096577 \\
ROA28 & 0,082638 & 0,164647 & 0,109967 & 0,092222 & 0,096577 \\
ROA37 & 0,082638 & 0,164647 & 0,109967 & 0,092222 & 0,096577 \\
ROA30 & 0,082638 & 0,164647 & 0,109967 & 0,092222 & 0,096577 \\
ROA6 & 0,082638 & 0,164647 & 0,109967 & 0,092222 & 0,096577 \\
ROA40 & 0,082638 & 0,164647 & 0,109967 & 0,092222 & 0,096577 \\
ROA3 & 0,082638 & 0,164647 & 0,109967 & 0,092222 & 0,096577 \\
ROA14 & 0,082638 & 0,164647 & 0,109967 & 0,092222 & 0,096577 \\
ROA5 & 0,082638 & 0,164647 & 0,109967 & 0,092222 & 0,096577 \\
ROA39 & 0,082638 & 0,164647 & 0,109967 & 0,092222 & 0,096577 \\
\hline
\end{tabular}

Tabela 4. Dados para a segunda requisição e pontuação com AHP

\begin{tabular}{|c|c|c|c|c|c|c|}
\hline \multirow{2}{*}{ ROA } & Re & Fo & Cl & Co & Me & \multirow{2}{*}{ Pont. } \\
\cline { 2 - 6 } & 0,09091 & 0,09091 & 0,23072 & 0,23072 & 0,52831 & \\
\hline R25 & 0,08263 & 0,16464 & 0,10996 & 0,32142 & 0,09222 & 0,17074 \\
R28 & 0,08263 & 0,16464 & 0,10996 & 0,32142 & 0,09222 & 0,17074 \\
R6 & 0,08263 & 0,16464 & 0,10996 & 0,32142 & 0,09222 & 0,17074 \\
R40 & 0,08263 & 0,16464 & 0,10996 & 0,32142 & 0,09222 & 0,17074 \\
R3 & 0,08263 & 0,16464 & 0,10996 & 0,32142 & 0,09222 & 0,17074 \\
R14 & 0,08263 & 0,16464 & 0,10996 & 0,32142 & 0,09222 & 0,17074 \\
R5 & 0,08263 & 0,16464 & 0,10996 & 0,32142 & 0,09222 & 0,17074 \\
R27 & 0,08263 & 0,16464 & 0,10996 & 0,32142 & 0,08111 & 0,16486 \\
R16 & 0,07654 & 0,14033 & 0,10996 & 0,32142 & 0,08111 & 0,16210 \\
R38 & 0,08263 & 0,03643 & 0,10996 & 0,32142 & 0,08111 & 0,15321 \\
\hline
\end{tabular}

Para a primeira, segunda e terceira requisições, foram utilizados os valores de pesos dos Indicadores para os respectivos critérios que os compõem e que foram requeridos pelo usuário. Assim, em todas as tabelas descrevendo os resultados dos experimentos, a segunda linha apresenta tais pesos. Da mesma forma, todas essas tabelas também apresentam os valores intermediários utilizados no cálculo da pontuação final, para os critérios envolvidos conforme sua compatibilização com o atendimento da requisição feita pelo usuário. Ainda, em todas essas tabelas, a posição ocupada na linha indica a classificação no ranqueamento de cada ROA. Assim, o primeiro ROA apresentado será o mais bem ranqueado, e assim sucessivamente.

Dessa forma, a Tabela 3, apresenta o resultado do primeiro experimento realizado, com a primeira requisição do usuário, considerada simples. Com ela, os dez primeiros ROAs classificados conseguiram atender $100 \%$ a requisição. Nesse caso, os 10 ROAs melhor classificados foram: ROA 25, ROA 28, ROA 37, ROA 30, ROA 6, ROA 40, ROA 3, ROA 14, ROA 5, ROA 39, respectivamente. Por meio de uma consulta a base de dados

\footnotetext{
${ }^{2}$ https://repositorios-de-objetos-de-aprendizagem.webnode.com/
} 
IX Congresso Brasileiro de Informática na Educação (CBIE 2020)

Anais do XXXI Simpósio Brasileiro de Informática na Educação (SBIE 2020)

Tabela 5. Dados para a terceira requisição e pontuação com AHP

\begin{tabular}{|c|c|c|c|c|c|c|c|c|c|c|}
\hline \multirow{2}{*}{ ROAs } & $\mathrm{Re}$ & $\mathrm{Fo}$ & $\mathrm{Cl}$ & $\mathrm{Ar}$ & $\mathrm{Cp}$ & $\mathrm{Na}$ & $\mathrm{Int}$ & $\mathrm{Ac}$ & $\mathrm{Me}$ & Pontuação \\
\cline { 2 - 10 } & 0,090 & 0,090 & 0,230 & 0,230 & 0,230 & 0,150 & 0,150 & 0,150 & 0,528 & \\
\hline ROA25 & 0,086 & 0,167 & 0,112 & 0,261 & 0,321 & 0,077 & 0,184 & 0,197 & 0,095 & 0,033 \\
ROA28 & 0,086 & 0,137 & 0,112 & 0,261 & 0,321 & 0,077 & 0,184 & 0,162 & 0,095 & 0,032 \\
ROA40 & 0,074 & 0,167 & 0,112 & 0,261 & 0,321 & 0,077 & 0,134 & 0,162 & 0,080 & 0,031 \\
ROA5 & 0,074 & 0,137 & 0,112 & 0,261 & 0,321 & 0,077 & 0,134 & 0,162 & 0,080 & 0,030 \\
ROA1 & 0,086 & 0,137 & 0,039 & 0,261 & 0,321 & 0,077 & 0,184 & 0,162 & 0,080 & 0,030 \\
ROA27 & 0,074 & 0,167 & 0,112 & 0,261 & 0,321 & 0,070 & 0,134 & 0,162 & 0,080 & 0,029 \\
ROA6 & 0,074 & 0,167 & 0,098 & 0,261 & 0,321 & 0,077 & 0,134 & 0,046 & 0,095 & 0,029 \\
ROA14 & 0,074 & 0,137 & 0,112 & 0,261 & 0,321 & 0,077 & 0,134 & 0,046 & 0,080 & 0,029 \\
ROA4 & 0,074 & 0,137 & 0,039 & 0,261 & 0,321 & 0,077 & 0,184 & 0,046 & 0,095 & 0,028 \\
ROA3 & 0,074 & 0,137 & 0,098 & 0,261 & 0,321 & 0,077 & 0,134 & 0,000 & 0,095 & 0,028 \\
\hline
\end{tabular}

é possível observar que esses ROAs tem características semelhantes em relação aos tipos de recursos, formatos de objetos de aprendizagem, funcionalidade interna dos ROA para colaboração e metadados.

Na segunda requisição, dos 10 ROAs classificados, sete (7) ROAs conseguiram atender $100 \%$ a requisição, portanto foram as alternativas melhor classificadas: ROA 25, ROA 28, ROA 6, ROA 40, ROA 3, ROA 14 e ROA 5 conforme mostra a Tabela 4. Assim, pode-se observar que para a primeira e segunda requisições, vários ROAs obtiveram o mesmo valor de pontuação. Sendo assim, o ranqueamento desses ROAs foi organizado de acordo com os valores da soma dos seus indicadores na base de dados.

Finalmente, a Tabela 5 apresenta o resultado da terceira requisição, em que somente o ROA 25 conseguiu atender inteiramente a requisição do usuário. Os demais ROAs conseguiram atender em partes, o que justifica suas posições no ranqueamento.

Nesses experimentos, pode-se observar que o método proposto trouxe com precisão qual o ROA é o mais adequado em termos de quantidade, por que encontra o ROA com mais recursos dentro de todo o conjunto, e com qualidade, uma vez que leva em consideração a preferência/necessidade do usuário em termos de Indicadores que se enquadram no âmbito da avaliação de qualidade de software, selecionando as alternativas de acordo com o que usuário deseja dos ROAs. Ainda, pode-se observar que conforme o número dos critérios e os valores utilizados nas requisições vão aumentando, os melhores ROAs se mantém sempre entre as 10 melhores posições no ranqueamento. É o que se observa em todas as requisições testadas, em que o ROA 25, ROA 28, ROA 40, ROA 6, ROA 14 e ROA 3 lá figuram. A diferença encontra-se nos valores da pontuação, que como esperado, para a primeira requisição pode-se observar que todos os ROAs atendendo a todos os critérios, possuem o mesmo valor. Porém, na segunda e terceira requisições nem todos se mantém com a mesma pontuação. Na segunda requisição somente sete ROAs possuem os mesmos valores de pontuação e na terceira somente o ROA 25 consegue atender.

\section{Considerações finais}

Esse trabalho propôs um método para avaliação de Repositórios de Objetos de Aprendizagem (ROA), tendo em vista Indicadores como: Funcionalidade, Usabilidade, Confia- 
bilidade e Compatibilidade. Foi utilizado o método multicritério para tomada de decisão AHP para realizar uma avaliação quantitativa e qualitativa para solucionar o problema de seleção e assim auxiliar na decisão de encontrar um ROA que melhor se adéqua a uma determinada necessidade do usuário. Foram utilizados valores reais para os Indicadores mencionados, coletados em 40 ROAs existentes e ativos.

Resultados da utilização do método comprovam sua funcionalidade, uma vez que dadas as requisições do usuário com seus resultados possíveis conhecidos de antemão, este é apresentado pelo método. Assim, a principal contribuição do trabalho consiste em criar uma forma especializada de avaliação de ROAs que inclui a requisição do usuário, uma vez que trabalhos correlatos avaliam ROAs utilizando apenas os valores de Indicadores. Como continuidade do trabalho, pretende-se realizar outras requisições para verificar o comportamento do método. A modelagem do problema utilizando outro método de auxílio a tomada de decisão, produzindo um método híbrido também é pensada como um passo futuro para o trabalho. Ainda, também pretende-se realizar uma comparação com métodos de avaliação de ROAs já existentes na literatura.

\section{Agradecimentos}

O presente trabalho foi realizado com apoio da Coordenação de Aperfeiçoamento de Pessoal de Nível Superior - Brasil (CAPES) - Código de Financiamento 001.

\section{Referências}

Braga, J. C. (2015). Objetos de Aprendizagem Vol.1: introdução e fundamentos, volume 1 of Intera. Editora da UFABC, Santo André, SP.

Cechinel, C., da Silva Camargo, S., Sicilia, M.-Á., and Alonso, S. S. (2016). Mining models for automated quality assessment of learning objects. J. UCS, 22(1):94-113.

Committee, L. T. S. et al. (2002). Ieee standard for learning object metadata. IEEE Standard, 1484(1):2007-04.

İnce, M., Yiğit, T., and Işık, A. H. (2019). A hybrid ahp-ga method for metadata-based learning object evaluation. Neural Computing and Applications, 31(1):671-681.

Kurilovas, E. (2013). Mceqls approach in multi-criteria evaluation of quality of learning repositories. In Innovations in XML Applications and Metadata Management: Advancing Technologies, pages 96-117. IGI Global, Hershey, PA.

Kurilovas, E., Vinogradova, I., and Kubilinskiene, S. (2016). New mceqls fuzzy ahp methodology for evaluating learning repositories: a tool for technological development of economy. Technological and Economic Development of Economy, 22(1):142-155.

Saaty, T. L. (1980). The analytic hierarchy process mcgraw-hill. New York, 324.

Standardization, I. O. (2016). Systems and Software Engineering: Systems and Software Quality Requirements and Evaluation (SQuaRE): Measurement of System and Software Product Quality. ISO, Geneva, Switzerland.

Yigit, T., Isik, A. H., and Ince, M. (2014). Multi criteria decision making system for learning object repository. Procedia-Social and Behavioral Sciences, 141:813-816. 